This item was submitted to Loughborough's Research Repository by the author.

Items in Figshare are protected by copyright, with all rights reserved, unless otherwise indicated.

\title{
Optimisation of high-speed crash avoidance in autonomous vehicles
}

PLEASE CITE THE PUBLISHED VERSION

http://dx.doi.org/10.1504/IJVAS.2012.051269

PUBLISHER

(c) Inderscience

VERSION

AM (Accepted Manuscript)

LICENCE

CC BY-NC-ND 4.0

REPOSITORY RECORD

Best, Matt C.. 2013. "Optimisation of High-speed Crash Avoidance in Autonomous Vehicles". figshare. https://hdl.handle.net/2134/11554. 
This item was submitted to Loughborough's Institutional Repository (https://dspace.lboro.ac.uk/) by the author and is made available under the following Creative Commons Licence conditions.

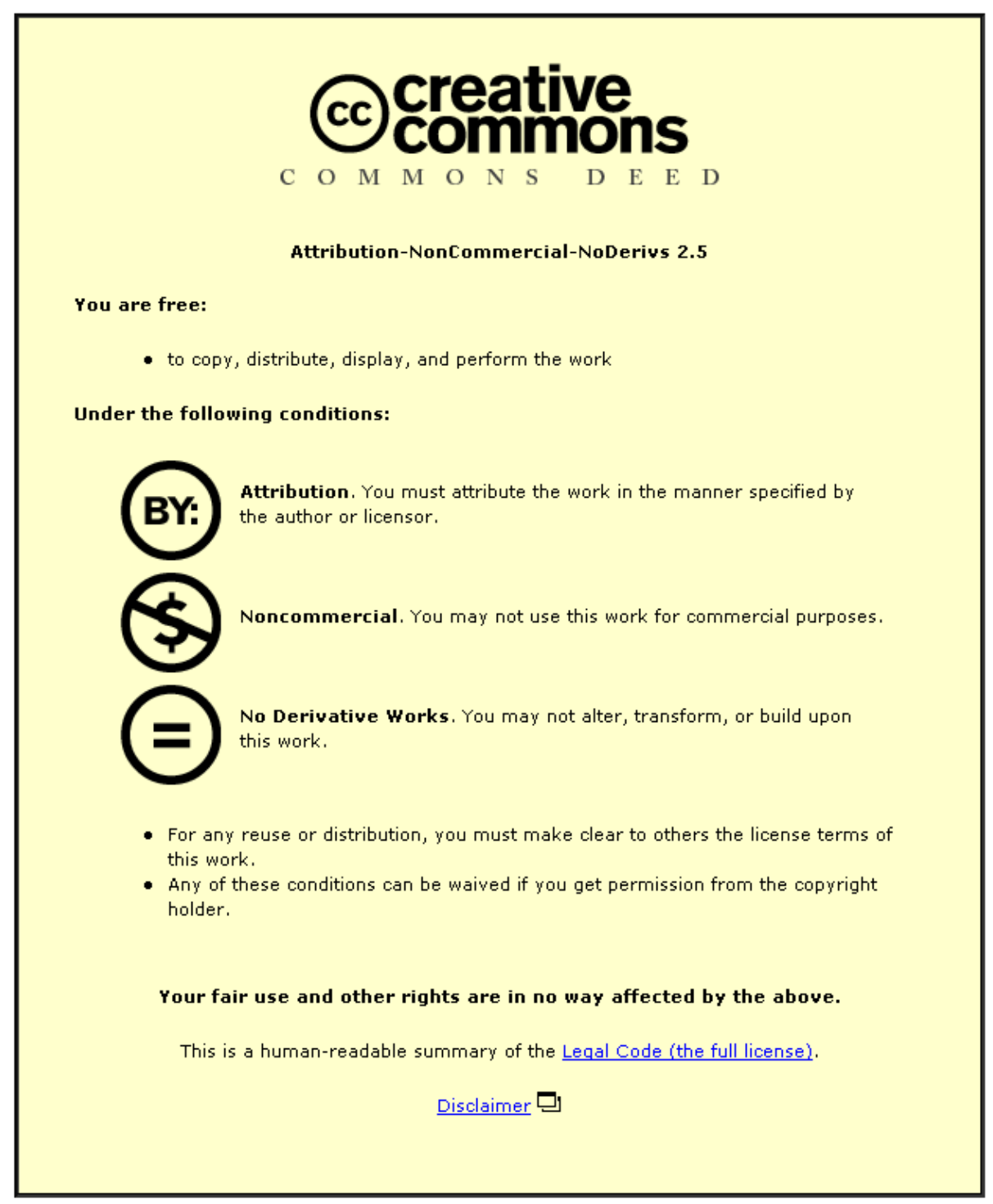

For the full text of this licence, please go to: http://creativecommons.org/licenses/by-nc-nd/2.5/ 


\title{
Optimisation of High Speed Crash Avoidance in Autonomous Vehicles
}

\author{
Matthew C Best \\ Senior Lecturer in Vehicle Dynamics and Control \\ Dept. Aeronautical and Automotive Engineering \\ Loughborough University \\ Leicestershire, UK \\ LE11 3TU \\ Email : M.C.Best@lboro.ac.uk \\ Phone : 01509227209 \\ Fax : 01509227275
}

\begin{abstract}
In the context of a future scenario of autonomous vehicle platooning, this paper considers the optimisation of a vehicle's standard brake, acceleration and steering control inputs, for collision avoidance. We consider the case where escape into the neighbouring lane is feasible. An iterative simulation based method is used, which allows vehicle parameters to be optimised simultaneously; this also allows us to find the best vehicle handling balance for such a manoeuvre, and to quantify the cost of suboptimal design. The paper also considers the relative advantages of speed reduction in conjunction with rapid lane change. The goal here is to quantify the best possible vehicle escape manoeuvre, and the relative cost of alternative strategies. The paper does not provide an immediately practicable controller, but simple openloop approximation of the optimal controls suggests a route towards future real-time solution.
\end{abstract}

Keywords: Vehicle Handling Dynamics, Optimisation, Obstacle Avoidance, Vehicle Design, Autonomous Vehicle

Biographical notes: Matt Best is a senior lecturer in vehicle dynamics and control in the department of Aeronautical and Automotive Engineering at Loughborough University in the UK. He gained his $\mathrm{PhD}$ in practical implementation of vehicle ride control at Loughborough in 1995, and has been a lecturer since 1996. His principal research interests include design of nonlinear optimal observers and controllers for vehicle ride and handling, Kalman filter methods for estimation and identification, vehicle dynamics modelling and real-time vehicle motion simulators. He has published approx. 40 papers in his field, in conferences, and journals such as International Journal of Vehicle Autonomous Systems, Proc. ImechE parts D and K, International Journal of Mechanics and Mobility, and International Journal of Vehicle Design.

\section{NOTATION}

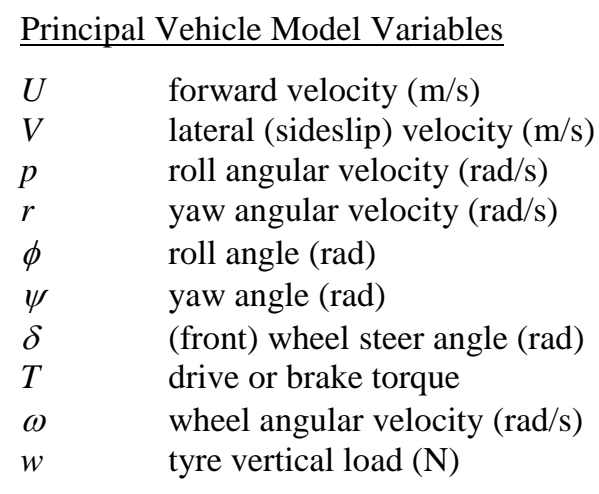

$\beta=-V / U$ vehicle body sideslip angle (degs)

Principal GOC Variables

$J \quad$ cost function

$L \quad$ continuous dynamic cost

$L_{T} \quad$ final, or residual cost

$\mathbf{x} \quad$ states vector

p costates vector

u inputs vector

$\mathrm{H}$ Hamiltonian function 


\section{INTRODUCTION}

The last decade has seen a huge increase in research into Advanced Driver Assistance Systems (ADAS), Autonomous Vehicles and Intelligent Transportation Systems (ITS). The availability of accurate GPS, wireless networks, cheaper proximity sensing and high speed vision processing all suggest that partial or total autonomy of traffic is a realistic mediumterm prospect. Prototype systems seen in programs such as PATH (Horowitz and Varaiya, 2000), DARPA (Ozguner et al 2007) and Prometheus (see Dickmanns 1999) illustrate that, while autonomy in urban and rough terrain conditions is difficult, Motorway driving presents perhaps the best application for autonomy. Great practical challenges with legislation and liability still pertain, but programmes such as PATH and Prometheus demonstrate that the technical challenges are nearer to solution. Also the often discussed advantages of autonomy - in transportation convenience, improved safety and road capacity relate most strongly to higher speed, multi-lane freeways controlled wholly or partly for platoons. This is the principal application environment of this paper, which focuses on collision avoidance within a high-speed environment.

The term 'collision avoidance' is rarely used in the literature in this context however. A search of Autonomous Vehicles and Collision Avoidance in one database returns over 600 papers from the last decade, where the vast majority concentrate on path planning for obstacle avoidance, with or without real-time path modification (eg Gehrig and Stein 2007, Liu et al 2006, Connors and Elkaim 2007). The manoeuvres are usually low speed and suited to robot manipulation, UGV and military applications. Those that do consider higher speed traffic scenarios typically impose acceleration limits within a comfortable lateral range for the occupant (eg Kwon et al 2005, Zhang and Ren, 2009) or within the wider feasible longitudinal range of the vehicle (eg Girault, 2004). This allows multiple vehicle interaction and traffic flow analysis considering interesting ITS scenarios, but it omits emergency manoeuvres and does not address vehicle handling dynamic limitations. More complex vehicle dynamic models are seen in Yoon et al (2009) and Borrelli et al (2005), but the former considers operation only at low speed and the latter assumes a fixed reference trajectory, with no avoidance manoeuvres.

The concept considered here is a high-speed emergency lateral avoidance manoeuvre, executed by an autonomous vehicle controller. As such it necessarily employs a full vehicle handling model incorporating dynamic roll behaviour and a combined-slip, load dependent nonlinear tyre model. The model is described in Section 2. A candidate (but not exclusive) application scenario is illustrated in Figure 1; a three-lane freeway has its outer two lanes devoted to an adjacent pair of platoons which maintain a 'chequerboard' configuration. In the event of an obstacle instantly appearing (such as a car crossing the central reservation, a rogue manually driven car cutting in, or an intrusion such as a pedestrian - even horses have been known in previous UK motorway incidents) affected vehicles in either autonomously controlled lane could very rapidly change to the other autonomous lane. If necessary, parts of the traffic (though presumably not the same vehicle) could change lane more than once if required. Undoubtedly, in such an extreme scenario, all affected traffic would also slow down in a coordinated way, but not necessarily by braking at the limit of tyre adhesion. The natural reaction of a human driver to an emergency is to brake harshly, and possibly also to steer; here we explore the optimal behaviour to achieve the required avoidance manoeuvre at the limit of the car's adhesion capacity - making better use of lateral capacity than longitudinal along with the most appropriate handling balance. 
As well as being more effective than a brake only strategy, the suggested behaviour lends itself to implementation alongside rapid real-time path prediction algorithms, such as those suggested in Lin et al (2000) and Batz et al (2009) (for the autonomous vehicles) and visual object tracking systems (for the obstacle). The rapid lane change also has applications beyond fully autonomous traffic systems, since in conjunction with suitable lateral proximity sensing it could be used as an ADAS, as an alternative to, or supplement to ABS.

The optimality of the avoidance manoeuvre is achieved by use of an iterative simulation based method known as Generalised Optimal Control (GOC). This has been employed effectively for ride and handling control optimisation (eg Gordon and Best 2002, Gordon and Best 2007). GOC establishes the optimal sequence of inputs to any smoothly nonlinear system that minimises any smooth cost function. It is a particularly attractive approach in this context as it allows simultaneous optimisation of time-varying controls (steering, acceleration and braking) and fixed model parameters. The collision avoidance scenario is also posed without the need for a reference vehicle path; the optimal path evolves as a result of appropriate cost functions on the obstacle, the vehicle position in the lane, and final stable vehicle orientation. The disadvantage of the technique is that it is very computationally expensive, and entirely unsuited to real-time implementation. This paper does not therefore arrive at an immediately practicable controller. Instead it establishes the feasible limit of obstacle avoidance behaviour of a vehicle, and the optimal sequences of input that achieve best performance.

Section 3 explains the general and specific applications of GOC. The simulation study of Section 4 then explores optimal steering in an evasive lane change with and without the addition of acceleration or braking. From this, the 'cost' of an additional speed reduction strategy is explored, and the study concludes with some considerations on practical implementation of the avoidance manoeuvre.

\section{VEHICLE MODEL}

The vehicle model is based on the well known three degree of freedom model, simulating yaw, roll, and sideslip using a load dependent, combined-slip Pacejka tyre model. A fourth, longitudinal degree of freedom is included, as are additional states for wheel-spin, and first order lags for tyre relaxation.

The principal equations of motion are

$$
\begin{array}{lc}
\text { longitudinal: } & M \dot{U}=\sum_{i=1,4} F_{x i}+M r V+M h r p \\
\text { lateral: } & M \dot{V}+M h \dot{p}=\sum_{i=1,4} F_{y i}-M U r \\
\text { yaw: } & I_{z z} \dot{r}=a \sum_{i=1,2} F_{y i}-b \sum_{i=3,4} F_{y i}+c \sum_{i=1,3} F_{x i}-c \sum_{i=2,4} F_{x i}
\end{array}
$$

roll:

$$
-I_{x z} \dot{r}+M h \dot{V}+I_{x x} \dot{p}=-M h U r-\left(B_{f}+B_{r}\right) p+\left(M g h-K_{f}-K_{r}\right) \phi
$$

roll kinematics:

$$
\dot{\phi}=p
$$


Standard SAE axes are used (Gillespie 1992) fixed relative to the vehicle wheelbase; the wheels are labelled (1-4) in ascending order as (front-left, front-right, rear-left, rear-right). The vehicle is initialised as a typical $\mathrm{C}$ class passenger car according to parameters suggested in (Dixon, 2004) - see Table 1.

The forces controlling the vehicle body motion $\left(F_{x i}, F_{y i}\right)$ allow for large steer angles

$$
\begin{array}{ll}
F_{x 1,2}=F_{t x 1,2}^{*} \cos \delta-F_{t y 1,2}^{*} \sin \delta, & F_{x 3,4}=F_{t x 3,4}^{*} \\
F_{y 1,2}=F_{t y 1,2}^{*} \cos \delta-F_{t x 1,2}^{*} \sin \delta, & F_{y 3,4}=F_{t y 3,4}^{*}
\end{array}
$$

based on lagged tyre forces, where each of the 8 elements are lagged to simulate relaxation within the tyre

$$
\dot{F}_{t x / y, i}^{*}=\tau^{-1}\left(F_{t x / y, i}-F_{t x / y, i}^{*}\right)
$$

and the tyre forces $\left(F_{t x i}, F_{t y i}\right)$ are modelled according to the Pacejka magic formula

$$
P(\mathbf{k})=P(\mathbf{k} ; B, C, D, E) \equiv D \sin \left(C \tan ^{-1}\left(B \mathbf{k}-E\left(B \mathbf{k}-\tan ^{-1} B \mathbf{k}\right)\right)\right)
$$

using normalized slip and isotropic similarity scaling (Milliken and Milliken 1995, Pacejka 2002). In more detail, the normalized slip vector is

$$
\mathbf{k}=\left(\begin{array}{l}
k_{x} \\
k_{y}
\end{array}\right)=\frac{C_{\alpha}}{F_{p}}\left(\begin{array}{c}
S \\
\tan \alpha
\end{array}\right)
$$

where $S$ is the longitudinal slip ratio, and $\alpha$ is the slip angle,

$$
S=\frac{r_{r} \omega-U_{x w}}{U_{x w}}, \quad \tan \alpha=\frac{-U_{y w}}{U_{x w}}
$$

based on wheel oriented velocities

$$
U_{x w}=U_{x} \cos \delta+U_{y} \sin \delta, \quad U_{y w}=U_{y} \cos \delta-U_{x} \sin \delta
$$

and

$$
U_{x 1,3}=U+c r, \quad U_{x 2,4}=U-c r, \quad U_{y 1,2}=V+a r, \quad U_{y 3,4}=V-b r
$$

The friction circle at each tyre contact patch is defined by a simple analytic function of vertical load $w$

$$
\sqrt{F_{t x}^{2}+F_{t y}^{2}} \leq F_{p}(w)=\frac{\mu w}{1+(w / M g)^{3}}
$$

and the load-dependent cornering/longitudinal stiffness for each tyre takes the form

$$
C_{\alpha}(w)=c_{1}\left(1-e^{-w / c_{2}}\right)
$$

so that the resulting tyre force vector is 


$$
\left(\begin{array}{l}
F_{t x} \\
F_{t y}
\end{array}\right)=P(|\mathbf{k}|) \frac{F_{p}}{|\mathbf{k}|}\left(\begin{array}{l}
k_{x} \\
k_{y}
\end{array}\right)
$$

Vertical tyre loads are calculated from static weight distribution, and modified to accommodate lateral load transfer according to :

$$
\Delta_{l a t} w_{f / r}=\frac{\sum F_{y f / r} h_{R}+K_{f / r} \phi+B_{f / r} p+M g h \sin \phi}{t_{f / r}}
$$

and longitudinal load transfer, according to

$$
\Delta_{\text {long }} w=\frac{\sum_{i} F_{x i} \cdot\left(h_{R}+h\right)}{(a+b)}
$$

Wheel rotational dynamics are modelled as

$$
\dot{\omega}_{i}=I_{w}^{-1}\left(T_{i}-r_{r} F_{t x i}\right)
$$

where the drive / brake torques $T_{i}$ are directly commanded and apportioned equally between left and right wheels, and in the case of brake torque, apportioned in the ratio 60:40 between front and rear axles. Drive torque is apportioned entirely to the front (FWD).

Finally in order to allow vehicle path based costs in the optimisation, CG position and orientation in global coordinates are found using the yaw angle,

$$
\begin{aligned}
& \dot{\psi}=r \\
& \dot{X}=U \cos \psi-V \sin \psi \\
& \dot{Y}=U \sin \psi+V \cos \psi
\end{aligned}
$$

[Table 1 here]

\section{SIMULATION BASED OPTIMISATION}

The optimisation is conducted using generalised optimal control (GOC). Controls are sought to minimise a Hamiltonian which is prescribed in terms of a (nonlinear) system of costate equations over a fixed time period.

\subsection{General formulation}

The method uses a gradient descent implementation of Pontryagin's Maximum Principle (see Bryson and Ho 1975 for the basic method, and Marsh 1992 for the application in automotive systems). GOC utilises a continuous dynamic cost function $L$ plus a residual cost associated with final vehicle states, $L_{T}$

$$
J=L_{T}[\mathbf{x}(T)]+\int_{0}^{T} L[\mathbf{x}(t), \mathbf{u}(t)] d t
$$


Adding constraint equations via a vector of Lagrange multiplier functions, $\mathbf{p}(t)$, yields

$$
J=L_{T}[\mathbf{x}(T)]+\int_{0}^{T}\left\{L[\mathbf{x}(t), \mathbf{u}(t)]+\mathbf{p}^{T}(t)[g[\mathbf{x}(t), \mathbf{u}(t)]-\dot{\mathbf{x}}(t)]\right\} d t
$$

where the function $g$ is defined via the nonlinear system equations

$$
\dot{\mathbf{x}}=g[\mathbf{x}(t), \mathbf{u}(t)]
$$

Lagrange multipliers (costates) $\mathbf{p}(t)$ are introduced and a Hamiltonian function is defined

$$
H=L[\mathbf{x}(t), \mathbf{u}(t)]+\mathbf{p}^{T}(t) g[\mathbf{x}(t), \mathbf{u}(t)]
$$

The costates are then chosen to satisfy the following differential equations

$$
\dot{\mathbf{p}}^{T}(t)=-\frac{\partial H}{\partial \mathbf{x}}=-\frac{\partial L}{\partial \mathbf{x}}-\mathbf{p}^{T} \frac{\partial g}{\partial \mathbf{x}}, \quad \mathbf{p}^{T}(T)=\frac{\partial L_{T}}{\partial \mathbf{x}}
$$

and the optimal controls are found from the Hamiltonian via

$$
\frac{\partial H}{\partial \mathbf{u}}=0
$$

Equations (22) - (25) are solved over the time interval $[0, T]$ assuming fixed initial states $\mathbf{x}(0)=\mathbf{x}_{0}$, and since the costate 'initial' conditions are set at $t=T$, the system is a two-point boundary value problem.

Following the methods used in (Marsh 1992, Best and Gordon 2002), an approximate solution is found via a discrete sequence of controls, each held constant for a small time $\Delta t$. Within the time period for each control, the cost gradient is obtained directly from the Hamiltonian

$$
\frac{\partial J}{\partial u_{i}}=\int_{t_{i-1}}^{t_{i}} \frac{\partial H}{\partial u_{i}} d t
$$

which then enables a gradient-based iterative optimisation to determine the optimal control sequence.

[Figure 2 here]

Figure 2 provides a summary of the algorithm used to conduct the GOC optimisation :

1 Using the current discrete control sequence, integrate the state-space system from $\mathbf{x}(0)$ and evaluate $J_{[0, T]}$

2 Evaluate the residual cost $L_{T}$ and hence $\mathbf{p}(T)$ from Equation (24)

3 Integrate the costate system and $\partial \mathrm{H} / \partial \boldsymbol{u}$ in reverse-time from the initial condition $\mathbf{p}(\mathrm{T})$. Calculate cost gradients from Equation (26)

4 Update the control sequence by a line search optimisation along the steepest descent direction to minimise $J$ (where $J$ is evaluated by repeating Stages $1 \& 2$ ).

Stages 1-4 are repeated until suitable convergence of cost and controls, and reduction of cost gradients is achieved. 


\subsection{Specific application}

The costate system of Equations (24) for the vehicle model is prohibitively complex to establish by hand, so three techniques are employed to create accurate, yet efficient simulation code. Firstly, the equations are manipulated using an analytical math processor - the Matlab $^{\mathrm{TM}}$ Symbolic toolbox. Direct evaluation of the partial derivatives is then possible, but the resultant formulae are long and inefficient (for example $\partial H / \partial u$ leads to an equation comprising over 100,000 characters). These direct formulae are thus only used to validate the final code, which is generated by first breaking each partial derivative into its component parts, eg

$$
\frac{\partial H}{\partial u}=\frac{\partial H^{\prime}}{\partial u}+\sum_{i} \frac{\partial H^{\prime}}{\partial F_{i}} \frac{\partial F_{i}}{\partial u}
$$

where $H^{\prime}$ is the Hamiltonian written in terms of the tyre forces $F_{i}$, and $\partial F_{i} / \partial u$ is further broken down in to component derivatives of the Pacejka formulae. The resulting derivatives are then converted into lines of computer code by an iterative extraction of common terms, to produce the shortest possible function.

To increase the efficiency of time integration of the states and costates, a discrete-time integration algorithm is employed; this is the Cash/Karp $5^{\text {th }} / 6^{\text {th }}$ order algorithm (Press et al, 1992). The time-step is kept constant within each control $\left(\mathbf{u}_{i}\right)$ time interval, and to ensure accuracy the code is written to monitor state errors and adjust the time-step durations accordingly.

Figure 2 illustrates that the control set can comprise controls varying at time intervals $\Delta t$ and also parameter controls which stay constant for the whole time interval $T$. The control set considered here is some combination of

$$
\mathbf{u}=\left[\begin{array}{llll}
\delta & u_{T d} & u_{T b} & \kappa
\end{array}\right]
$$

where acceleration and braking are determined via

$$
T_{i}=\left(u_{T d}^{2}-u_{T b}^{2}\right) M
$$

and $\kappa$ is a parameter control, initialised at zero, affecting roll moment distribution, via

$$
K_{f}=K_{f 0}+1000 \kappa, \quad K_{r}=K_{r 0}-1000 \kappa
$$

The time interval $\Delta t$ is set as $0.01 \mathrm{~s}$ here; the precise choice is not significant, provided the interval is sufficiently small relative to the system dynamics.

The cost function is required to be continuous along any reasonable state trajectory, and here this is dominated by a track cost which is illustrated in Figure 3. The philosophy is that the shaded danger areas force an initial deviation of at least $2.5 \mathrm{~m}$ (a large truck width) in as short a distance in $X$ as possible, and that recovery should not allow the vehicle to pass outside the safe right hand lane. The fillet section $2<Y<2.5$ uses a half cosine to ensure a smooth transition of cost between high and zero cost regions.

A small final cost ensures that the vehicle ends the simulation in the centre of the safe right hand lane, aligned with the road, and with no significant yaw rate or sideslip velocity : 


$$
L_{T}=\rho_{\psi} \psi^{2}+\rho_{r} r^{2}+\rho_{v} v^{2}+\rho_{Y}(Y-3.7)^{2}
$$

Each of the $\rho$ coefficients are set to give equal cost, based on a preliminary optimisation, and then scaled to ensure a total cost contribution below $0.1 \%: \rho_{\psi}=325, \rho_{r}=200, \rho_{v}=8, \rho_{Y}=$ 200.

The final continuous cost function also includes elements which ensure feasible inputs and vehicle behaviour :

$$
L=L_{\text {track }}+\lambda_{u} u^{2}+\lambda_{\beta} \beta^{2}+\lambda_{\delta} \delta^{2}
$$

where various combinations of the $\lambda$ coefficients are considered in Section 4. 


\section{SIMULATION EXPERIMENTS}

\subsection{Unconstrained Optimisation}

Consider first a relatively unconstrained optimisation of the vehicle, controlled by steering alone, and with $\kappa$ simultaneously optimised. The conditions are

$$
\mathbf{u}=\left[\begin{array}{ll}
\delta & \kappa
\end{array}\right] \quad \lambda_{u}=\lambda_{\beta}=\lambda_{\delta}=0
$$

The results in Figure 4 show how the cost has converged, over 12000 iterations, with two intermediate points illustrated alongside the final solution. Note how the steer strategy (plot b) has changed dramatically through the optimisation, even though plot c shows that peak tyre force is achieved and exceeded throughout the manoeuvre; the GOC method is capable of convergence even in the presence of uncontrollable and unstable behaviour in single tyres. In addition to the distinct cost saturation seen after 9000 iterations in plot a, final convergence is also confirmed by reduction in the cost gradients, $\partial J / \partial u_{i}$, and further iterations see only a further reduction of cost gradients with cost unvaried. The GOC method is successful, but slow; the 12000 iterations required to optimise $\delta$ and $\kappa$ together, at the limit of the tyres, takes several hours - an overnight run on a conventional PC with $2.66 \mathrm{Ghz}$ processor.

The optimal behaviour is extreme, and indeed physically unrealisable. Peak steer angles exceed $85^{\circ}$ and plot $\mathrm{d}$ shows high body sideslip up to $25^{\circ}$; later results are constrained to achieve realistic peak $\delta$. In all optimisations including $\kappa$, the same interesting result is seen; the vehicle seeks high roll stiffness at the front, and zero at the rear. In fact, $\kappa=14.3$ is the largest achievable passive redistribution of stiffness possible for this vehicle, so the optimal results here are imposing the equivalent of active suspension forces to minimise rear load transfer, and hence maximise rear lateral forces.

[Figure 4 here]

\subsection{The Vehicle Dynamics of Optimised High Speed Lane-change}

Constraints are now applied, and realistic results obtained to explore the vehicle dynamics of the avoidance manoeuvre, and the relative merits of allowing high front roll stiffness and/or acceleration or braking in combination with steering to achieve lower cost and faster escape behaviour. Here

$$
\lambda_{u}=0, \quad \lambda_{\beta}=40, \quad \lambda_{\delta}=30
$$

are nominally chosen, to impose an additional cost on the extreme sideslip and steer values seen in Section 4.1 (about 10\% of total cost if those levels were retained). Input conditions and final cost comparisons are given in Table 2, where we also see 'headway limit' values; these are the distance along the road at which the vehicle first achieves a CG lateral deviation of $+2.5 \mathrm{~m}$ (the distance along the road at which an obstacle such as a large stationary truck is avoided). Note that a distance, rather than time metric is used, since the precise scenario is unknown and it is assumed that the controlled vehicle's speed contributes significantly to the danger.

[Table 2 here] 
Although we see that high $\kappa$ reduces headway in all cases, and the addition of acceleration or braking improves cost and headway, the range of headway limits between best and worst is only $1.3 \mathrm{~m}$. In all cases, steered avoidance is more than twice as effective as braking only, at the limit. Figure 5 shows that the optimal lane-change is still a very severe manoeuvre; in spite of the increased costing, peak sideslip still exceeds $20^{\circ}$, though this is now achieved with a feasible steer input, which peaks for the worst case at $34^{\circ}$. Higher $\lambda_{\beta}$ would reduce peak $\beta$, but only at significant cost in increased headway, as we will see in Section 4.4.

It is interesting that all cases invoke essentially the same vehicle behaviour. The strategy starts with high right steer to induce high yaw and lateral deviation, but also to reduce vehicle speed. After rear tyre forces have developed (plot d) to peak lateral accelerations of around $7 \mathrm{~m} / \mathrm{s}^{2}$, (not shown) an almost step change to even higher left steer at 0.75 seconds results in saturation of the rear axle, which is caught by right counter-steer applied in the non-braking cases at around 1.3 seconds. This counter-steer is adjusted to recover stability over the next second; this is clear from the vehicle sideslip between 1.2 and 2.5 seconds. Further steering corrections complete the stabilisation within the safe lane. The path for all cases remains similar to that seen in plot $4(d)$.

\section{[Figure 5 here]}

Case 4 achieves a better result than case 1, by minimising lateral load transfer, and hence maximising lateral force capacity at the rear. This enables case 4 to maintain the high initial steer input (plot a) whereas the standard vehicle must reduce steer at 0.5 seconds to prevent rear saturation and associated loss of control. Although front axle capacity is reduced in case 4, this has little detrimental effect in the manoeuvre, since the front axle is severely oversaturated in any case, to achieve deceleration as well as lateral acceleration.

Interestingly, this combined function of the front axle is retained when braking is added as a control, in case 3. Only a short braking input is used in the first few tenths of a seconds (plot a); this reduces speed slightly, but is then removed to prevent longitudinal forces reducing lateral force capacity at the front axle. With braking, case 3 inserts an additional stage in the vehicle behaviour between 0.75 and 1.5 seconds, where the rear lateral forces are diminished (plot d) allowing the rear to drift out to a larger peak sideslip angle with the left steer now acting as counter-steer. After this intermediate drift (during which the wheels do not lock not shown) the brakes are released and again a higher negative sideslip peak occurs which is recovered using an even higher impulsive brake application.

For case 2, drive torque is applied (to the front wheels) in phase with the onset of the front axle lateral force, and in combination with slightly higher right steer initially. By driving the front wheels with high steer, the more heavily loaded outer wheel contributes to the yaw moment, but the drive torque also reduces longitudinal load transfer to the front axle. The higher lateral forces that can develop at the rear in the first second (plot d) limit the reduction in headway caused by maintaining higher speed. Also notable is the use of acceleration in combination with steering to catch the instability that develops after steering left, allowing the highest peak $\beta$ of all cases.

\subsection{The Cost of Speed Reduction}

Table 2 shows that case 3 returns the shortest headway, and also delivers a useful reduction (of over 30\%) in speed (Figure 5b). Although not the principal aim, speed reduction will improve outcome in the event of a collision, so further reductions in speed are a useful 
consideration. To this end, Figure 6 considers a comparison between case 3 and an optimisation with the same actuators and cost function, but with the speed cost $\lambda_{u}=0.06$ set nominally to make speed account for about one quarter of total cost.

The resulting time histories are very similar to those seen in Section 4.2 and we see the same strategy, though with extended periods of braking, in Figure 6. The speed reduction is modest however; the final speed in case 3 is $20.5 \mathrm{~m} / \mathrm{s}$ and here it is $19.2 \mathrm{~m} / \mathrm{s}$. Cost has increased to 437.3 - an increase almost identical to the cost of $\lambda_{u} u^{2}$ integrated over the three seconds of the test - showing that little difference in vehicle response has been achieved. Headway has increased, marginally, to 31.6. The simple conclusion is that very little further reduction in speed is possible, because the complex destabilisation and recapture of the vehicle is still necessary for the lateral motion. Of equal concern however, is that to achieve the modest speed reduction, the new optimisation requires some very rapid reductions and reversals of steer around 2 seconds. It also generates even higher peak vehicle slip angles.

[Figure 6 here]

\subsection{Approximating the Emergency Lane-change with Open-loop Control}

Although it is not impossible to conceive of a feedback controller which could approximate the extreme escape manoeuvre shown here, it is sensible to be sceptical about the feasibility of balancing complex control commands to both steer and accelerator / brake, at high frequencies, to achieve the very best performance seen in Section 4.3 and in cases 2 and 3 in Section 4.2. In this final section, a simple feasibility experiment is carried out; the steer only case 1 optimisation is compared with two simple alternative open-loop steer sequences chosen to illustrate a) controller feasibility, and b) the cost of vehicle stability :

a) The feasible step steer input approximates the optimised steer with similar magnitude, using steps $\delta=+17^{\circ},-34^{\circ},+17^{\circ}$. The timing of the steps was tuned approximately to recover stability in the vehicle, and achieve an acceptable CG path.

b) The stable sine steer input uses a sinusoidal input, starting with high positive steer (ie phased as cosine) with magnitude maximised $\left(3^{\circ}\right)$ such that the vehicle remains stable throughout, and with frequency set $(0.4 \mathrm{~Hz})$ to achieve an acceptable $\mathrm{CG}$ path.

Both open-loop sequences were filtered through a $1^{\text {st }}$ order lag with time constant 0.05 seconds, to further ensure actuator bandwidth feasibility. (Optimisation of the inputs was conducted with the filter in the loop.)

The results in Figure 7 show that both open-loop controls were successful, though both need better trajectory control within the safe right hand lane. The step steer has achieved lower vehicle sideslip, at a cost of less than $2 \mathrm{~m}$ in headway. However the cost of imposing stability at all times in the vehicle seems large, with almost $10 \mathrm{~m}$ conceded in headway for the sine steer case. An appropriate route to designing a practical real-time controller would be to couple the destabilising step-steer open-loop inputs, probably scaled according to estimated road friction, with a suitable closed-loop yaw controller to recover vehicle stability in the safe lane.

[Figure 7 here] 


\section{CONCLUDING REMARKS}

In an emergency obstacle avoidance scenario at $70 \mathrm{mph}$, braking alone, even with perfect optimisation of tyre slip, will not always be effective. If we can assume reasonable accuracy in object tracking and path planning functions of a future autonomous vehicle, avoidance is theoretically possible via lane-change in less than half the distance along the road.

The optimal rapid lane-change is an aggressive, high g manoeuvre, which destabilises the vehicle; much of the control effort is assigned to restabilisation after a high initial steer which generates high yaw moment and also speed reduction. Interestingly, only a small reduction in headway distance results from adding brake or acceleration control, or altering the handling balance in the vehicle. A combination of brake and steer control has some value in speed reduction, but the controller design challenges of using braking as well as steer within the critical first two seconds of the manoeuvre may be too great. The optimal handling balance is extreme understeer, which will not be acceptable during normal, manual driving; small increases in understeer do not significantly alter outcomes for the high speed lane-change.

This study has established the performance capacity of a theoretical vehicle; the technique can not be applied directly to a test vehicle, due to the very high computational requirement of GOC, and the assumed perfect knowledge of the tyre and friction coefficient. A possible route towards identifying practical real-time control has been explored however; simple openloop control simulations suggest that steer only control should be feasible, though clearly any viable implementation would employ closed loop control - probably of yaw rate - to restabilise the vehicle. If the vehicle is not destabilised, the best alternative rapid lane-change may require $30 \%$ more headway.

In conclusion, provided headway clearance can accurately be identified in real-time, and road friction and tyre capacity are adequately known, the scope exists for extremely rapid lanechange which would only be used in exceptional circumstances, but which may prove valuable in avoiding high speed collisions.

\section{REFERENCES}

Batz, T., Watson, K. and Beyerer, J. (2009) 'Recognition of Dangerous Situations Within a Cooperative Group of Vehicles', IEEE Intelligent Vehicles Symposium, pp 907-912

Best, M.C. and Gordon, T.J. (2002) 'Simultaneous Optimisation of Vehicle Parameter and Control Action to Examine the Validity of Handling Control Assumptions', Proceedings of the 6th International Symposium on Advanced Vehicle Control (AVEC), Hiroshima, Japan, pp 87-92.

Borrelli, F., Falcone, P., Keviczky, T., Asgari, J. and Hrovat, D. (2005) 'MPC-based Approach to Active Steering for Autonomous Vehicle Systems', International Journal of Vehicle Autonomous Systems, vol 3, no 2-4, pp 265-291

Bryson A.E. and Ho, Y.C. (1975) 'Applied Optimal Control: Optimisation, Estimation and Control', Hemisphere, New York.

Connors, J. and Elkaim, G. (2007) 'Analysis of Spline Based, Obstacle Avoiding Path Planning Algorithm', IEEE Vehicular Technology Conference, pp 2565-2569

Dickmanns, E.D., (2002) 'Vision for Ground Vehicles: History and Prospects', International Journal of Vehicle Autonomous Systems, vol 1, no 1, pp 1-44

Dixon, J.C. (2004) 'Tires, Suspension and Handling', SAE International 
Gehrig, S.K. and Stein, F.J. (2007) 'Collision Avoidance for Vehicle-following Systems', IEEE Transactions on Intelligent Transportation Systems, vol 8, no 2, pp 233-244

Gillespie, T.D. (1992), 'Fundamentals of Vehicle Dynamics', SAE International

Girault, A., (2004) 'A Hybrid Controller for Autonomous Vehicle Driving on Automated Highways', Transportation Research Part C : Emerging Technologies, vol 12, no 6, pp 421-452

Gordon, T.J. and Best, M.C., (2002) 'A Sequential Dual Model Approach to Lap Optimisation', Proceedings of the 6th International Symposium on Advanced Vehicle Control (AVEC), Hiroshima, Japan, pp 99-104

Gordon, T.J. and Best, M.C., (2007) 'On the Synthesis of Driver Inputs for the Simulation of Closedloop Handling Manoeuvres', International Journal of Vehicle Design, vol 40, no 1-3, pp 52-76

Horowitz, R. and Varaiya, P., (2000) 'Control Design of an Automated Highway System' Proceedings of the IEEE, vol 88, no 7, pp 913-925

Kwon, S-J., Fujioka, T., Cho, K-Y. and Suh, M-W. (2005) 'Model-Matching Control Applied to Longitudinal and Lateral Automated Driving', Proceedings of the Institution of Mechanical Engineers, Part D: Journal of Automobile Engineering, vol 219, no 5, pp 583-598

Liu, D.K., Wu, X., Kulatunga, A.K. and Dissanayake, G. (2006) 'Motion Coordination fo Multiple Autonomous Vehicles in Dynamics and Strictly Constrained Environments', IEEE Conference on Cybernetics and Intelligent Systems, DOI : 10.1109/ICCIS.2006.252342

Lin, C-F., Ulsoy, A.G. and LeBlanc, D.J. (2000) 'Vehicle Dynamics and External Disturbance Estimation for Vehicle Path Prediction', IEEE Transactions on Control Systems Technology, vol 8, no 3, pp 508-518

Marsh C., (1992) 'A Nonlinear Control Design Methodology for Computer-controlled Vehicle Suspension Systems', PhD Thesis, Loughborough University.

Milliken, D.L. and Milliken, W.F. (1995) 'Race Car Vehicle Dynamics', SAE International

Ozguner, U., Stiller, C. and Redmill, K. (2007) 'Systems for Safety and Autonomous Behavior in Cars: The DARPA Grand Challenge Experience', Proceedings of the IEEE, vol 95, no 2, pp $397-412$

Pacejka, H.B. (2002) 'Tyre and Vehicle Dynamics', Butterworth Heinemann

Press, W.H. Teukolsky, S.A. Vetterling W.T. and Flannery, B.P. (1992), 'Numerical Recipes : The Art of Scientific Computing', Cambridge University Press, Cambridge.

Yoon, Y., Shin, J., Kim, H.J., Park, Y. and Sastry, S. (2009) 'Model-Predictive Active Steering and Obstacle Avoidance for Autonomous Ground Vehicles', Control Engineering Practice, vol 17, no 7, pp 741-750

Zhang, J-M. and Ren, D-B (2009) 'Lateral Control of Vehicle for Lane Keeping in Intelligent Transportation Systems', International Conference on Intelligent Human-Machine Systems and Cybernetics, vol 1, pp 446-450 


\begin{tabular}{|ll|}
\hline$I_{x x}$ & body roll moment of inertia $\left(400 \mathrm{kgm}^{2}\right)$ \\
$I_{z z}$ & yaw moment of inertia $\left(1900 \mathrm{kgm}^{2}\right)$ \\
$I_{x z}$ & roll/yaw product of inertia $\left(0 \mathrm{kgm}^{2}\right)$ \\
$I_{w}$ & wheel moment of inertia $\left(1.2 \mathrm{kgm}^{2}\right)$ \\
$M$ & vehicle mass $(1175 \mathrm{~kg})$ \\
$a$ & longitudinal Distance of C of G to front axle $(1.16 \mathrm{~m})$ \\
$b$ & longitudinal Distance of C of G to rear axle $(1.42 \mathrm{~m})$ \\
$h$ & CG height above roll axis $(0.44 \mathrm{~m})$ \\
$h_{R}$ & height of roll axis above ground at CG $(0.18 \mathrm{~m})$ \\
$c$ & front / rear half track $(0.72 \mathrm{~m})$ \\
$r_{r}$ & tyre rolling radius $(0.3 \mathrm{~m})$ \\
$K_{f 0}$ & front roll stiffness $(27.6 \mathrm{kNm} / \mathrm{rad})$ \\
$K_{r 0}$ & rear roll stiffness $(14.3 \mathrm{kNm} / \mathrm{rad})$ \\
$B_{f}$ & front roll damping $(1.55 \mathrm{kNms} / \mathrm{rad})$ \\
$B_{r}$ & rear roll damping $(1.14 \mathrm{kNms} / \mathrm{rad})$ \\
$B, C, D, E$ & Pacejka formula coefficients $(0.71,1.4,1.0,0.0$ - dimensionless $)$ \\
$c_{0}, c_{2}$ & cornering stiffness parameters $(37.2 \mathrm{kN} / \mathrm{rad}, 3.33 \mathrm{kN})$ \\
$\mu$ & road surface friction coefficient $(0.8)$ \\
$\tau$ & time-constant for tyre relaxation $(0.05 \mathrm{sec})$ \\
\hline
\end{tabular}

Table 1. Vehicle Model Parameter Values 


\begin{tabular}{|c|c|c|c|}
\hline case & Input conditions & cost & Headway limit (m) \\
\hline 1 & $\mathbf{u}=[\delta] \quad \kappa=0$ & 350.9 & 32.77 \\
\hline 2 & $\mathbf{u}=\left[\begin{array}{ll}\delta & T_{d}\end{array}\right] \quad \kappa=0$ & 321.0 & 32.06 \\
\hline 3 & $\mathbf{u}=\left[\begin{array}{ll}\delta & T_{b}\end{array}\right] \quad \kappa=0$ & 320.8 & 31.52 \\
\hline 4 & $\mathbf{u}=[\delta] \quad \kappa=14.3$ & 332.9 & 32.08 \\
\hline 5 & $\mathbf{u}=\left[\begin{array}{ll}\delta & T_{d}\end{array}\right] \quad \kappa=14.3$ & 318.4 & 31.78 \\
\hline \multirow[t]{3}{*}{6} & $\mathbf{u}=\left[\begin{array}{ll}\delta & T_{b}\end{array}\right] \quad \kappa=14.3$ & 317.2 & 31.47 \\
\hline & $\begin{array}{l}\text { Unconstrained optimisation (Section 4.1) } \\
\qquad \mathbf{u}=[\delta] \quad \kappa=0\end{array}$ & $\begin{array}{l}\text { Not } \\
\text { comparable }\end{array}$ & 31.75 \\
\hline & $\begin{array}{l}\text { Straight line braking alone, at the limit, to rest } \\
\qquad \mathbf{u}=\left[T_{b}\right] \quad \kappa=0\end{array}$ & $\begin{array}{l}\text { Not } \\
\text { comparable }\end{array}$ & 66.12 \\
\hline
\end{tabular}

Table 2. Cost and Headway Comparisons 


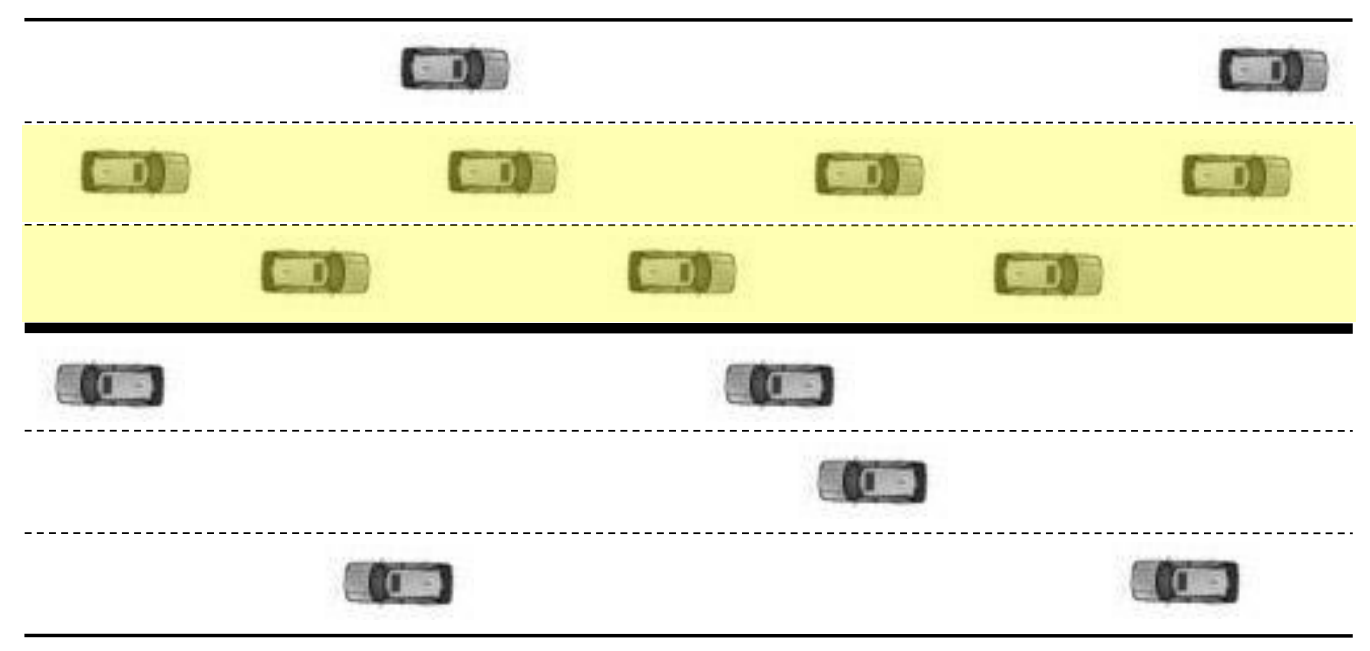

Figure 1: 'Chequerboard' Platooning Scenario (highlighted) 


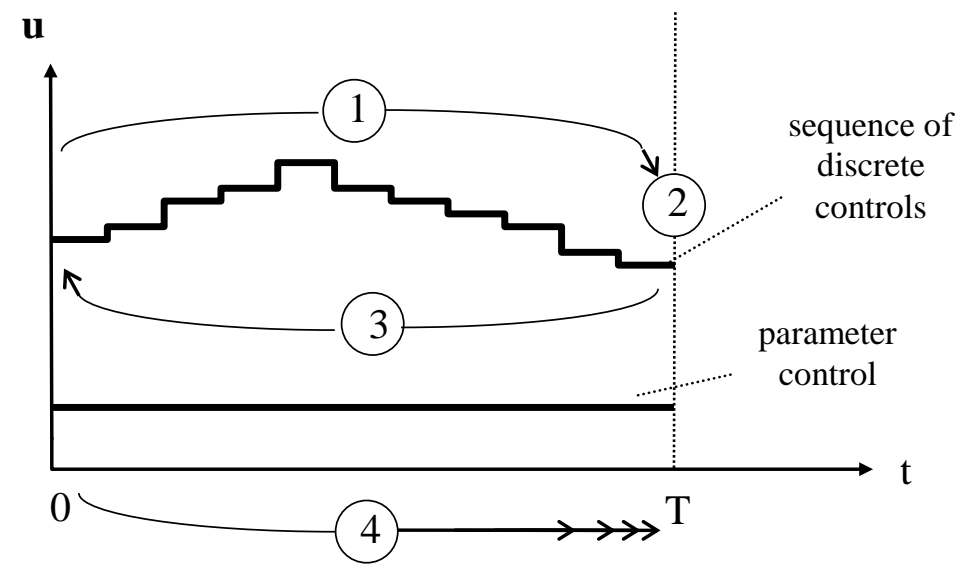

Figure 2 : Summary of the GOC Algorithm 


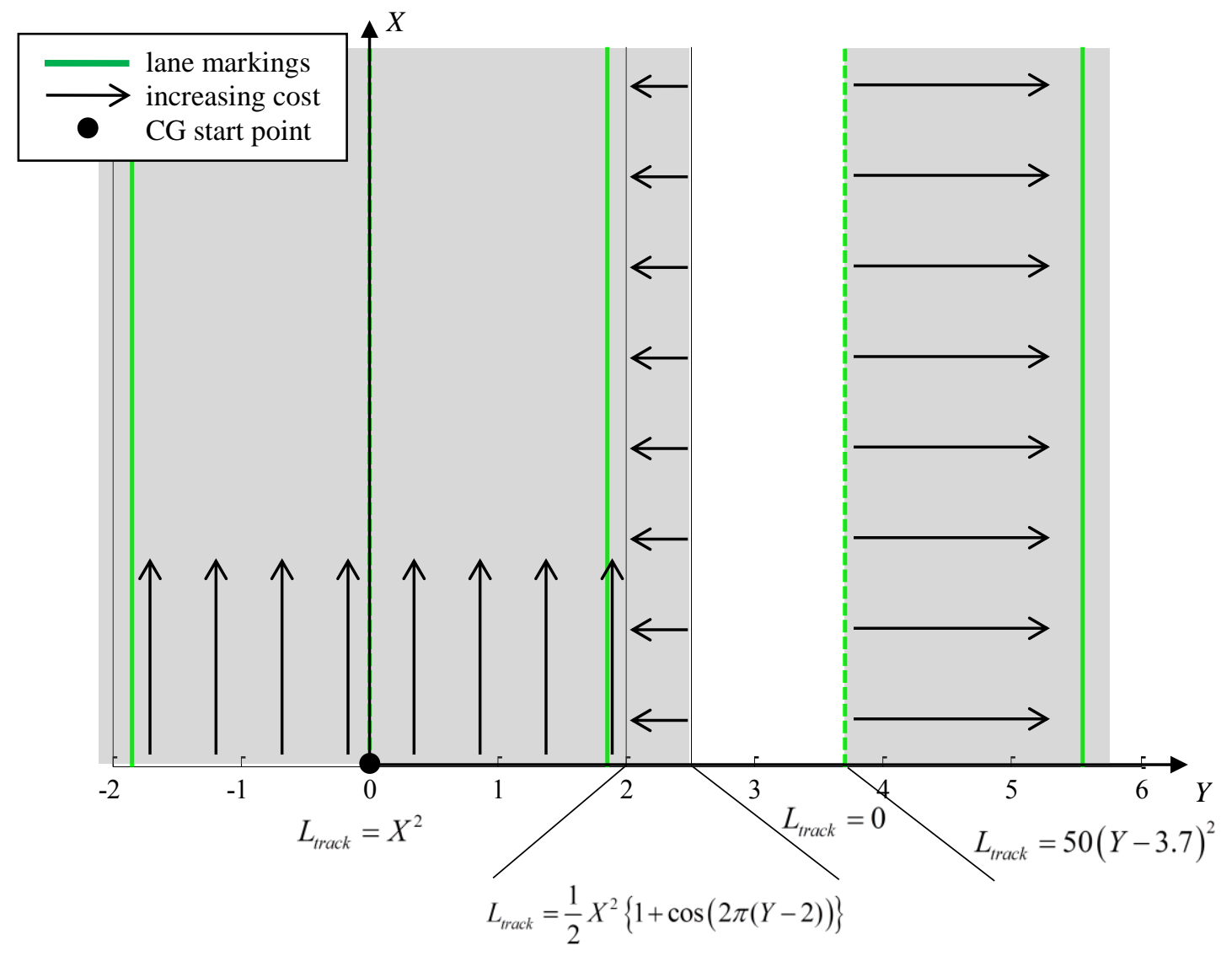

Figure 3 : Track cost topology 

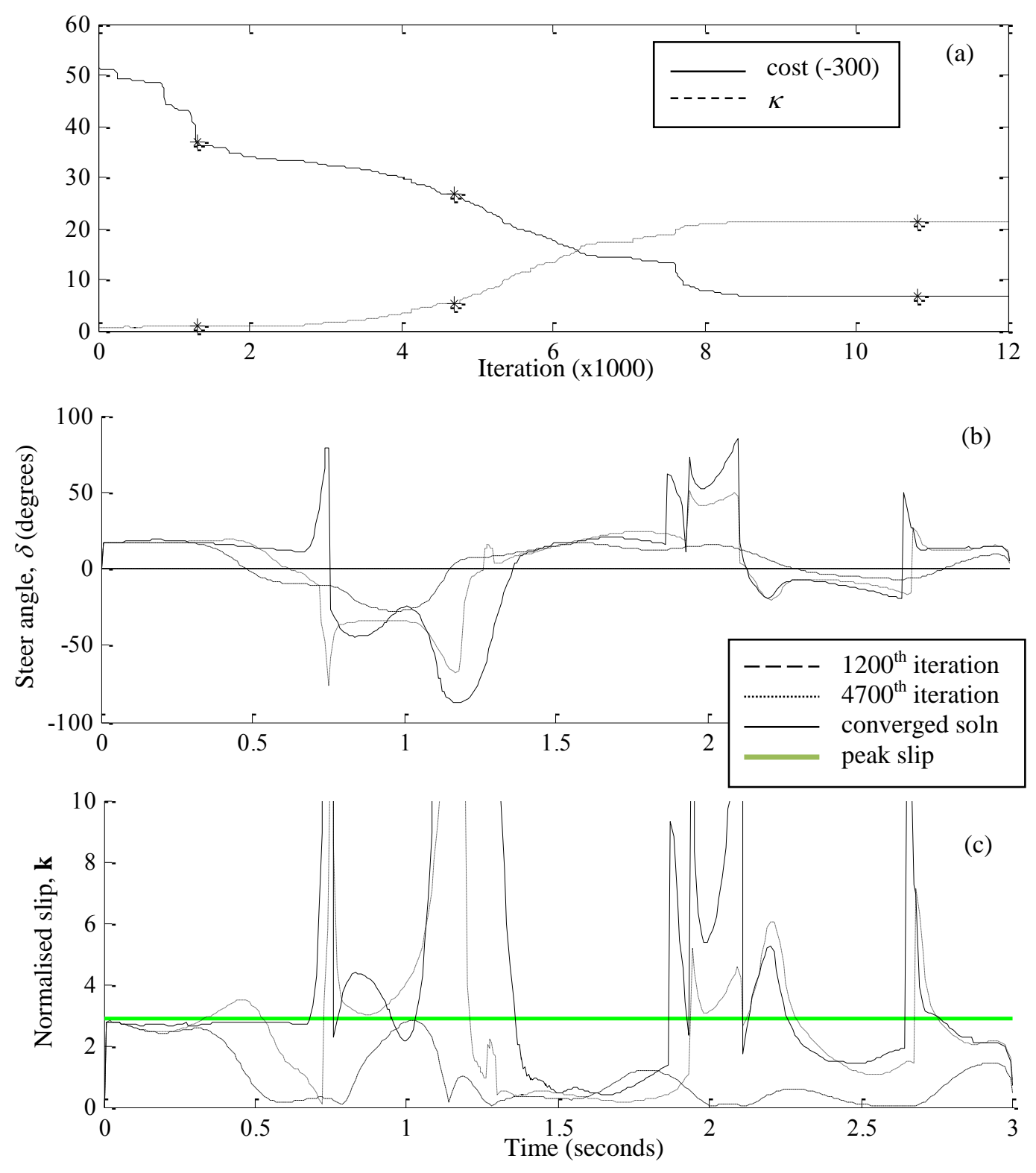

Figure 4 : Unconstrained Optimisation

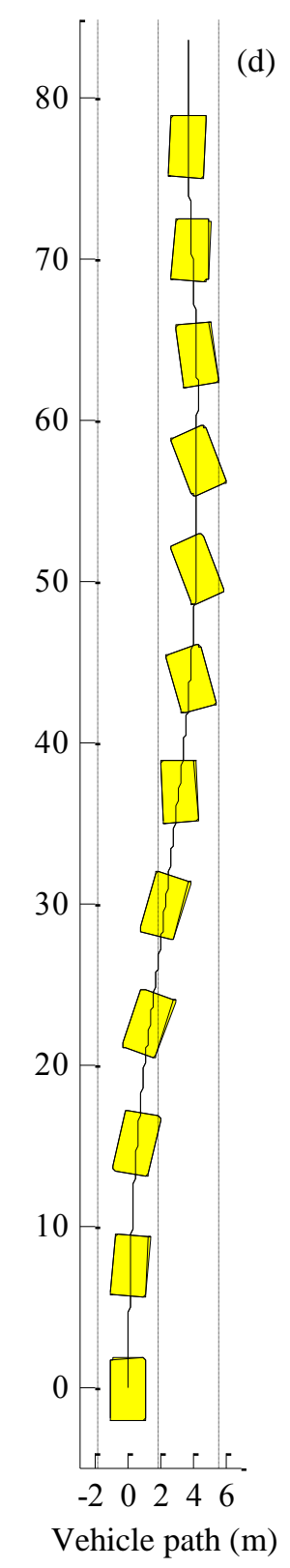



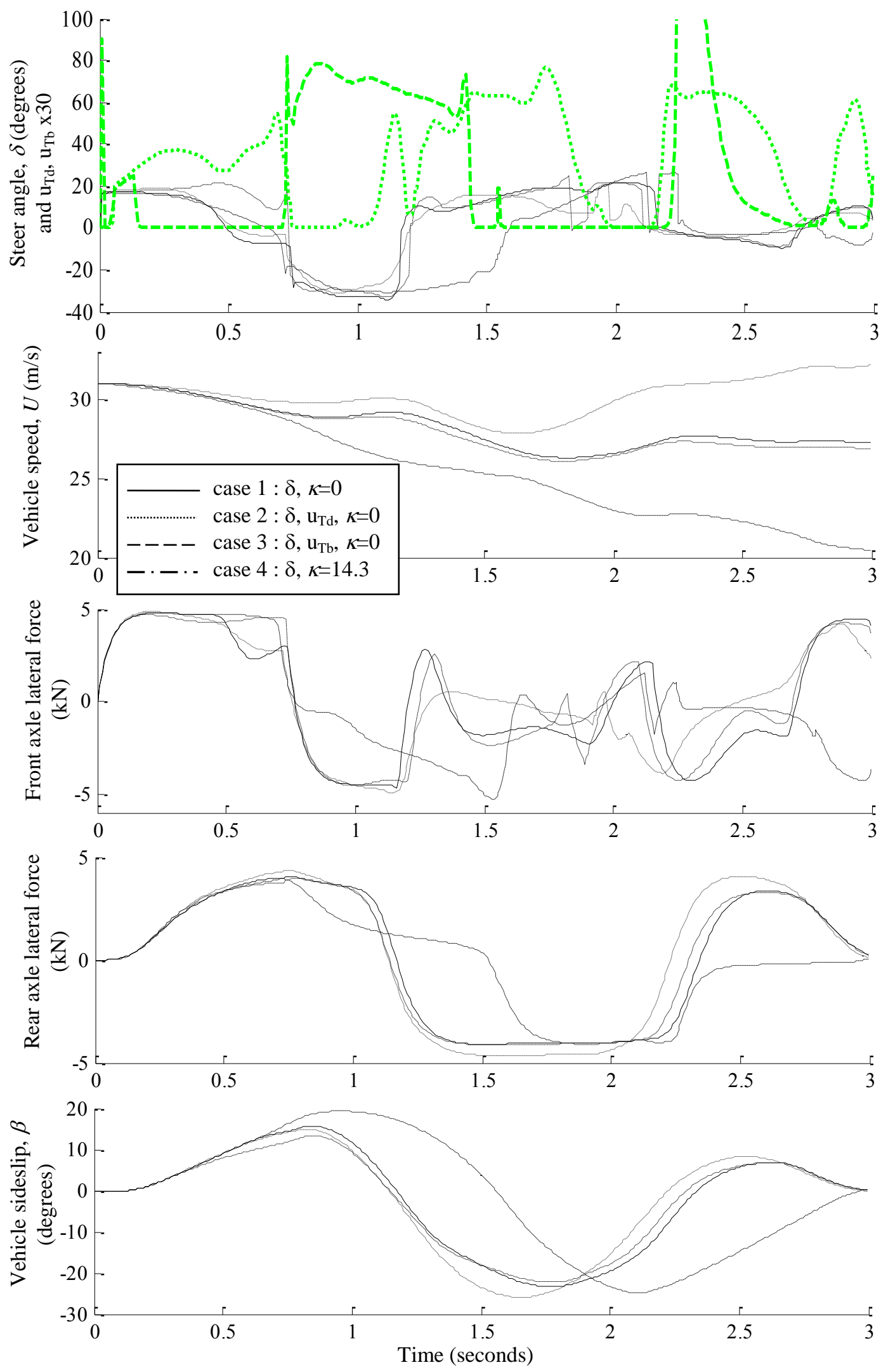

Figure 5 : Vehicle Dynamics Comparison 


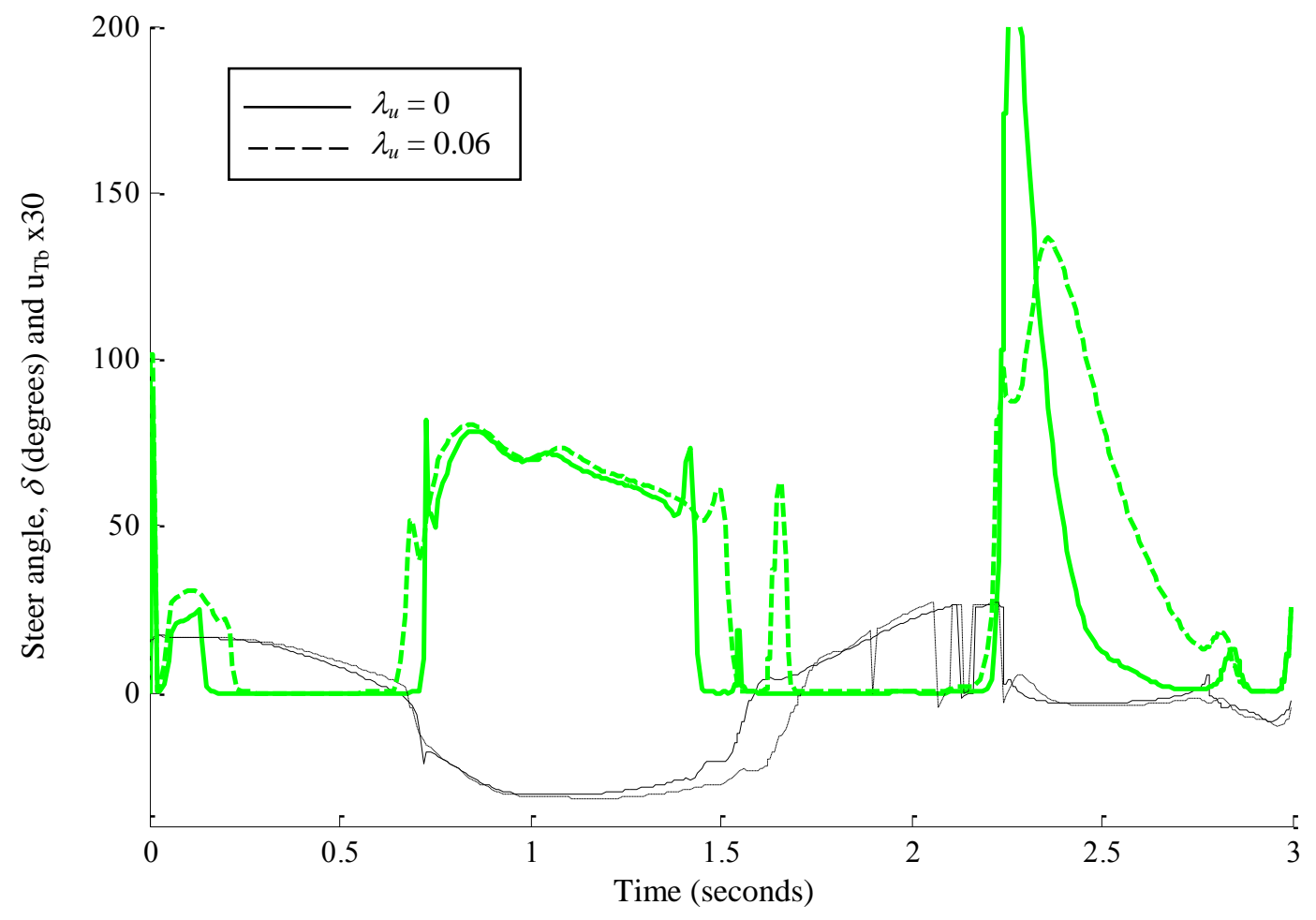

Figure 6 : The Cost of Speed Reduction 

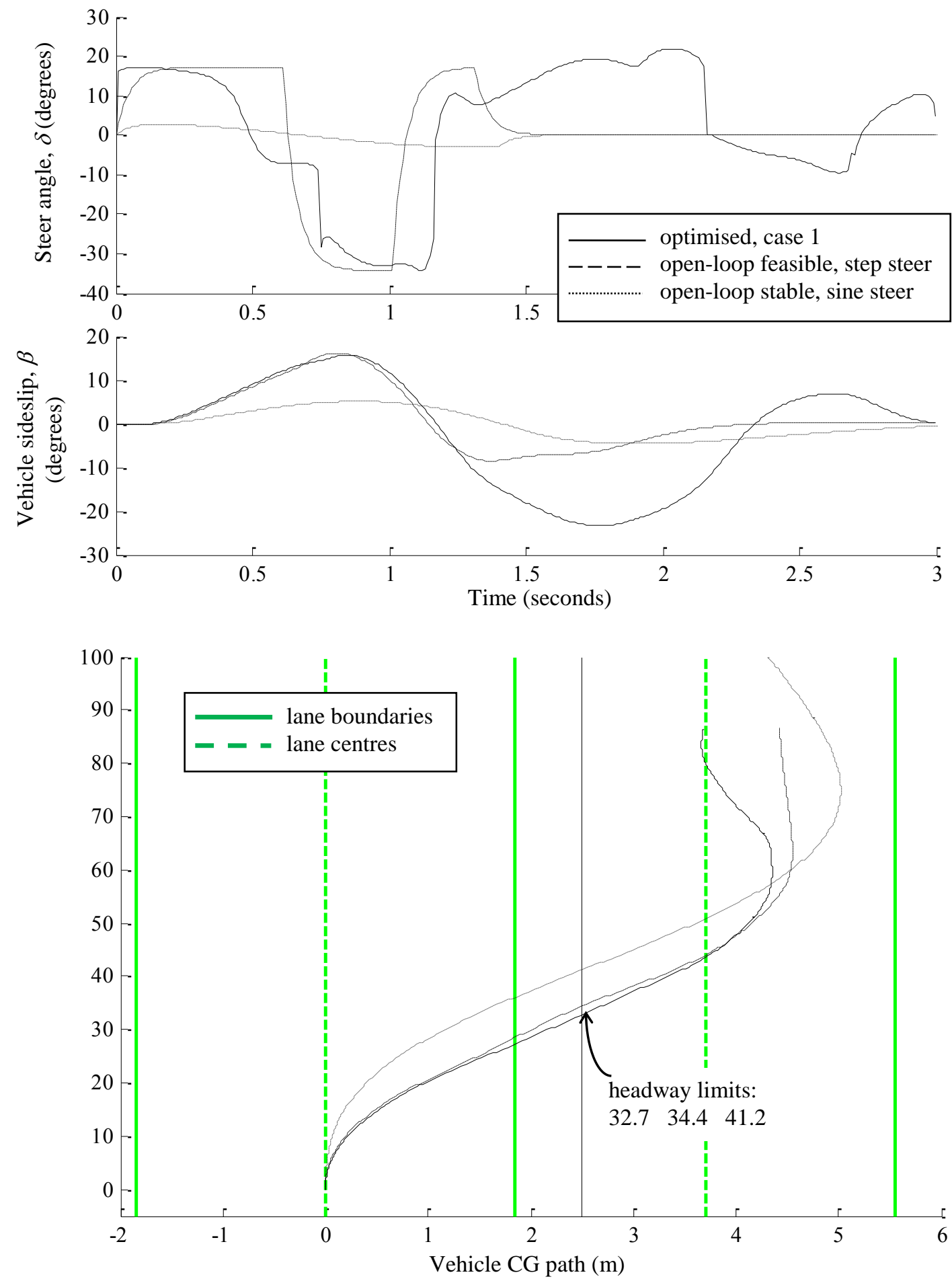

Figure 7 : Comparing Optimised with Open-loop Control Strategies 